LA-UR- $02-4793$

Approved tor public release; distribution is unlimited.
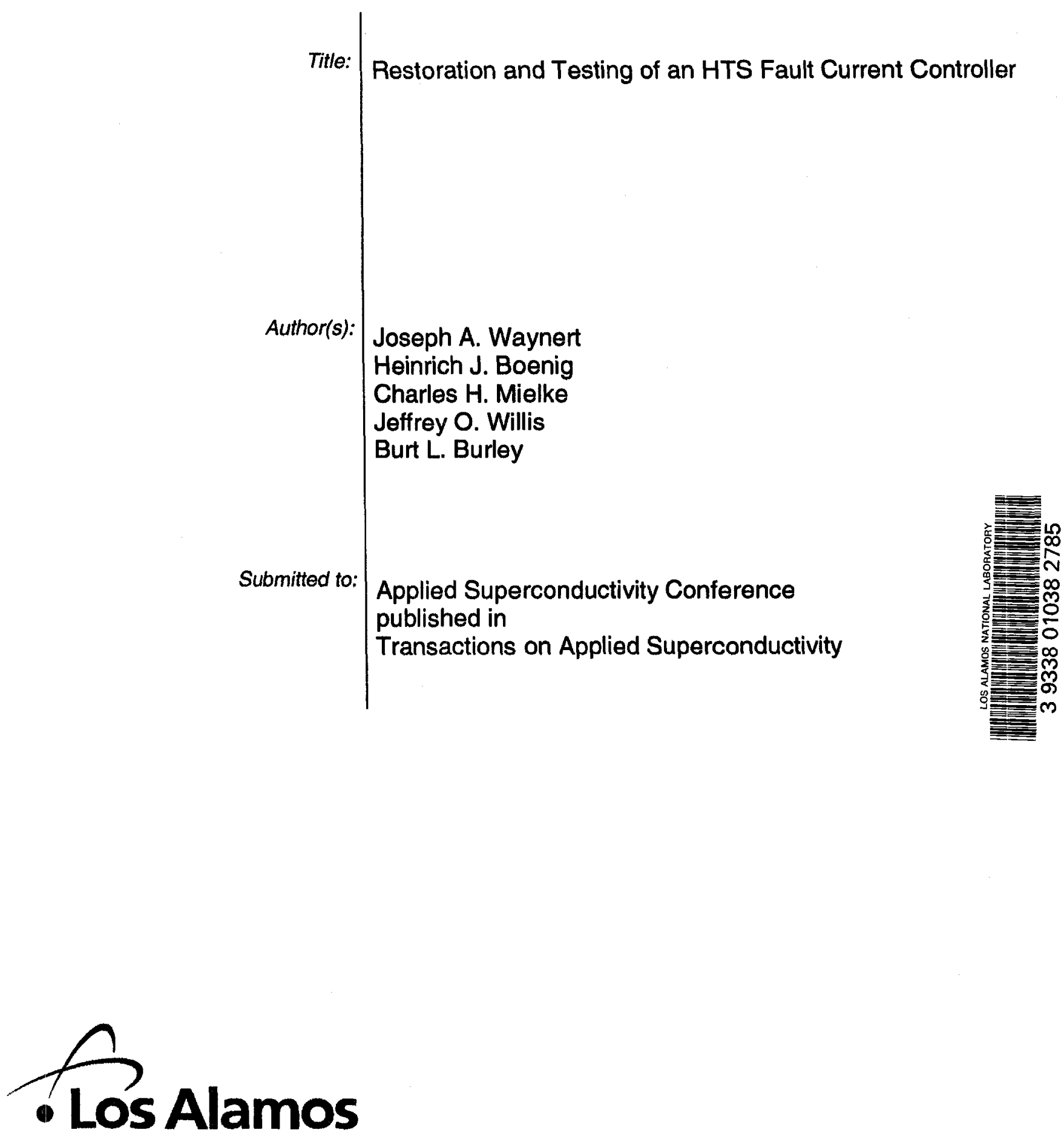

NATIONAL LABORATORY

Los Alamos National Laboratory, an affirmative action/equal opportunity employer, is operate $\equiv$ he University of Callfornia for the U.S. Department of Energy under contract W-7405-ENG-36. By acceptance of this article, the put $\nabla . r$ recognizes that the U.S. Government retains a nonexclusive, royalty-free license to publish or reproduce the published form of this contribution, or to allow others to do so, for U.S. Government purposes. Los Alamos National Laboratory requests that the publisher identify this article as work performed under the auspices of the U.S. Department of Energy. Los Alamos National Laboratory strongly supports academic freedom and a researcher's right to publish; as an institution, however, the Laboratory does not endorse the viewpoint of a publication or guarantee its technical correctness. 


\title{
Restoration and Testing of an HTS Fault Current Controller
}

\author{
Joseph A. Waynert, Senior Member, IEEE Heinrich J. Boenig, Member, IEEE, Charles H. Mielke, \\ Jeffrey O. Willis, Member, IEEE, Burt L. Burley
}

\begin{abstract}
A three-phase, $1200 \mathrm{~A}, 12.5 \mathrm{kV}$ fault current controller using three HTS $4 \mathrm{mH}$ coils, was bult by industry and tested in 1999 at the Center Substation of Southern California Edison in Norwalk, CA. During the testing, it appeared that each of the three single-phase units had experienced a voltage breakdown, one externally and two internally. Los Alamos National Laboratory (LANL) was asked by DOE to restore the operation of the fault current controller provided the HTS coils had not been damaged during the initial substation tests. When the internally-failed coil vacuum vessels were opened it became evident that in these two vessels, a flashover had occurred at the high voltage bus section leading to the terminals of the superconducting coil. An investigation into the failure mechanism resulted in six possible causes for the flashover. Based on these causes, the high voltage bus was completely redesigned. Single-phase tests were successfully performed on the modiffed unit at a $13.7 \mathrm{kV}$ LANL substation. This paper presents the postulated voltage flashover failure mechanisms, the new high voltage bus design which mitigates the failure mechanisms, the sequence of tests used to validate the new design, and finally, the results of variable load and short-circuit tests with the single-phase unit operating on the LANL $13.7 \mathrm{kV}$ substation.
\end{abstract}

Index Terms-current controller, dielectric breakdown, fault current limiter, fault current controller, high temperature superconductor

\section{INTRODUCTION}

$\mathrm{T}$ HIS project is a follow-on to a Department of Energy (DOE) Superconductivity Partnership Initiative (SPI) to develop a $15 \mathrm{kV}$ class 26 MVA high temperature superconducting (HTS) Fault Current Controller (FCC) [1].

Manuscript received August 5, 2002. This work was supported in part by the U.S. Department of Energy Office of Power Technologies, as part of the Superconductivity Program for Electric Systems.

J. A. Waynert is with Los Alamos National Laboratory, Los Alamos, NM 87545 USA (corresponding author phone: 505-667-6843; fax: 505-665-7740; $\mathrm{e}-$ mail: waynert@ lanl.gov).

H. J. Boenig is retired from Los Alamos National Laboratory, Los Alamos, NM 87545 USA (325 W Burbank, Fredericksburg, TX 78624).

C. H. Mielke is with Los Alamos National Laboratory, Los Alamos, NM 87545 USA (e-mail: cmielke@lanl,gov).

J. O. Willis is with Los Alamos National Laboratory, Los Alamos, NM 87545 USA (e-mail: jwillis@ lanl.gov).

B. L. Burley is with General Atomics, San Diego, CA 92121 USA (e-mail: burt.burley@gat.com).
The final stage of the initial SPI involved installation and testing of the FCC in the Center Substation of Southern California Edison (SCE). High voltage breakdown in each of the three phases of the FCC rendered the device inoperable and precluded the completion of the demonstration tests. DOE then contracted with Los Alamos National Laboratory (LANL) to evaluate the extent of the damage, and if feasible, repair or modify the FCC, and perform high voltage testing at the $13.7 \mathrm{kV}$ substation at Los Alamos.

This paper presents the potential causes of the high voltage breakdown, and the high voltage (HV) bus design changes which address these potential failure mechanisms. Finally, we present the very successful results of the single-phase testing of a modified FCC vessel, which demonstrated many of the capabilities of an HTS FCC.

\section{PRINCIPLe of Operation}

A fault current limiter (FCL) is a device placed in an electric network to limit the peak current in the event of a fault. Many projects are aimed at developing a resistive type HTS FCL. In this type device, the HTS element is placed in series with the circuit breaker. When a fault occurs, the increasing current exceeds the critical current of the HTS element causing a rapid increase in impedance in the circuit, which limits the value of the fault current.

In contrast, the FCC is based on a full-wave bridge. The four thyristors as shown in Fig. 1 provide the blocking and current limiting features. The HTS coil remains superconducting under normal operating conditions. A dc bias power supply establishes a bias current through the four thyristors with half the coil current passing through either of the two legs. The ac load current is superimposed on the bias current. If the peak ac current exceeds the magnitude of the dc bias current, as in a short circuit, the current value will pass through zero, turning off a pair of thyristors each half cycle. The inductance is automatically switched into the circuit, limiting the current because of the added impedance. With the additional capability to control the phase of the firing of the thyristors, the magnitude of the ac current can be regulated from the maximum shorting value to zero. Hence, the FCC can control the value of the fault current rather than merely limit it [2]. 


\section{HV BUS FAILURE MECHANISMS AND MITIGATION}

\section{A. Failure Mechanisms}

As mentioned above, the FCC has a vacuum vessel with an HTS coil for each of the three electrical phases. The vessel provides the cryostat for maintaining the temperature and thermal isolation of the HTS coil. Three GM cryocoolers are used to cool the coil, the thermal shield, and the HV leads. The compressors are electrically isolated from the cold heads.

One of the vessels had experienced a small internal helium leak and was being actively pumped during the testing at the SCE substation. That vessel experienced HV breakdown through the residual gas in the polymer tubing connecting the backing pump to the diffusion pump mounted on the vessel. Upon disassembly of the other two vessels, evidence of burn marks on the vacuum vessel, vaporization of multi-layer insulation (MLI), and partial melting of HV bus bolt threads was seen. It appeared that an electrical arc had passed from the HV bus to a nearby, grounded cold head.

Some of the more obvious causes of the HV breakdown were field enhancement by the presence of sharp corners, and the presence of dirt particles. Other less obvious potential causes of the breakdown were identified:

1. The HTS coil may see twice the applied voltage because of a resonance between the source reactance and the stray capacitance of the coil. A low inductance capacitive snubber across the coil could remedy this.

2. The source also has a stray capacitance, which, combined with the coil stray capacitance, can result in fast (nanosecond time scale) charging currents in the bus. The increased joule heating from the currents within the skin depth of the bus could evaporate any condensate on the bus. This would raise the gas pressure locally, decreasing the breakdown voltage.

3. The MLI near the HV bus penetration of the thermal shield appeared unconstrained. The MLI is readily displaced by an electric field which could arc to the material, vaporizing it, producing a local gas cloud, reducing the discharge strength, and allowing an arc from the bus to the grounded region.

4. A current in the bus generates a local magnetic field, which forces any free electrons in the vacuum space to undergo a helical motion. There may be a two-order decrease in the magnitude of the breakdown voltage when the diameter of the helical motion becomes comparable to the mean free path of residual gas [3], [4]. For the FCC, this would occur for a current of about 20 $\mathrm{kA}$ as might be generated per item 2 above.

\section{B. Mitigation Approach}

The more obvious causes of the HV breakdown listed above can be avoided through general HV engineering practice. The redesigned bus has no sharp corners. Furthermore, all assembly was done in a clean environment with efforts taken to minimize the production and agitation of dust. All the internal components of the FCC vessels received a final cleaning with ethanol using lint-free materials to remove dust particulates. Three of the other four voltage breakdown mechanisms can be mitigated with a simple change in design of the bus. The HV conductor is now a Litz wire (individually insulated multistrand conductor) inside a stainless steel tube. The tube is wrapped in a dielectric and a grounding sheath is applied as a final wrap. Thus, the electric field is confined to the annular space between the inner stainless tube and the ground sheath. The two exceptions to the confined electric field design are the warm end of the bus where it connects to the HV bushing which penetrates the vacuum vessel top plate, and the opposite, cold end of the bus which interfaces to the cryocooler. Finite element electrostatics analysis was used to minimize electric field enhancements in these two regions. Convoluted end fittings were used to lengthen the HV tracking paths connecting the ground sheath termination to the HV portion of the bus. Fig. 2 illustrates the main features of the modified bus.

\section{HV Bus and Cold Head Interface}

Although the internal HV breakdown occurred between the bus and the coldhead, it was recognized that the next likely failure location was at the interface between the HV bus and the coldhead. Fig. 2 shows an aluminum nitride (AlN) disk separating the HV bus from the cryocooler coldhead. The AlN acts as an electrical insulator isolating the bus from the grounded coldhead, and as a thermal short, allowing heat to be transferred from the bus to the coldhead.

The dielectric strength of AlN as given by the vendor, Kyocera, is $12 \mathrm{kV} / \mathrm{mm}$, and should be more than adequate given the $15 \mathrm{~mm}$ thickness separating the bus and the coldhead. The main concern is surface flashover. A mock-up of the coldhead, AlN, and HV bus was fabricated. A series of experiments were performed to determine the breakdown voltage as a function of temperature and vacuum pressure. The detailed results are presented in [5]. Several design modifications were suggested. These were initially evaluated using a finite element electrostatics code. Two configurations were tested. The installed configuration used a dielectric varnish to cover the triple junction between the AlN, the vacuum, and a copper disk of the same diameter as the AlN and in contact with the HV bus. During hipot testing of the installed configuration, breakdowns which were not selfhealing began to occur at $15 \mathrm{kV}$. The goal was $26 \mathrm{kV}$, but it was decided that this performance was adequate for testing in the LANL substation where the maximum applied voltage would be less than $5 \mathrm{kV}$.

\section{Single-Phase Substation Tests}

Fig. 3 shows the circuit that was established to test the 
FCC. The LANL substation, shown to the left of the vertical dotted line, includes the $4 \mathrm{mH}$ line inductance and a master breaker (MB). The components outside the substation may be disconnected and grounded from the substation through the D/GS blade switch. A $2.3 \mathrm{mH}$ protection inductor was required by the utility to limit the maximum current that could be drawn. For load tests, the FCC is connected in series with a $64 \Omega$ neutral grounding resistor that draws 1 MW of power. For load step tests, the Jennings switch (JS) is closed so that the load increases to $3 \mathrm{MW}$. For shorting tests, the Westinghouse breaker (WB) is closed to short the load.

\section{A. Voltage Withstand}

After verification of the control and operation of all the breakers and switches, the first test of the FCC was a voltage withstand test. With the thyristors in a blocking mode, the full voltage was applied to the FCC to verify that the electronics could successfully withstand the voltage for an extended time.

\section{B. Load and Load Step}

For proper operation, the FCC should be transparent to the system during normal loading and load changes. To test this, a representative $1 \mathrm{MW}$ resistive load was applied. The ac load current, the current through the thyristors, and the voltages at selected points were measured. The test would also demonstrate that the bias power supply was not necessary. To simulate load changes, the system was started with the $1 \mathrm{MW}$ load, then switched to a $3 \mathrm{MW}$ load, and finally, switched back to the $1 \mathrm{MW}$ load.

Fig. 4 shows representative results of a load step test. The $60 \mathrm{~Hz} 175 \mathrm{Aac}$ peak load current for the $1 \mathrm{MW}$ load switches to 525 Aac during the $3 \mathrm{MW}$ operation and back to 175 Aac as the load switches back to $1 \mathrm{MW}$. Also shown is the nearly dc current through the HTS coil. Without the bias power supply, the coil bias current is drawn from the power source. The bias current adjusts itself to equal the amplitude of the load current. Once the bias current is established, the ac current flows unimpeded through the FCC, except for two short time periods during the $60 \mathrm{~Hz}$ cycle when the bias current tends to become smaller than the load current and has to be recharged to the peak ac value. The coil current decrease is based on the time constant determined by the coil inductance and the series resistance of the coil and thyristors. There is also a forward voltage drop across the thyristors, which creates an additional effective resistance. The cyclic decay and recharge of the bias current is the ripple seen in Fig. 4.

\section{Fault, and Current Control}

The FCC was tested for fault response by energizing the 1 MW load and then shorting across the load by closing the Westinghouse breaker, as discussed above. If the thyristors are triggered at the zero crossing of the current, the FCC coil will be in the ac circuit for the minimum duration and the ac current will be a maximum. However, the ac current may be reduced through use of phase-delay angle control. The triggering of the thyristors can be delayed by a phase angle; the longer the phase delay, the longer the FCC coil remains in the ac circuit. The FCC acts as a variable inductance with values from zero to infinite with the corresponding ac current varying from a maximum value to zero. Fig. 5 illustrates one example of the FCC responding to a fault imposed at about $0.18 \mathrm{~s}$ with the phase delay angle set at 120 degrees. Note the change in scale for the ac current compared to Fig. 4. The large, nearly $4200 \mathrm{~A}$, peak current half cycle is the maximum ac current without the FCC coil in the circuit, and evidence of the time required for the control system to react. Fig. 6 shows the peak ac fault current as determined by the setting of the phase delay angle.

\section{Conclusions}

The key features of a bridge-type FCC have been demonstrated with single-phase testing in a $13.7 \mathrm{kV}$ substation. The unit consists of power electronic switching devices with their associated cooling, and an HTS coil cooled by three GM cryocoolers. The main features demonstrated during testing were the current limiting, current regulation via phase-angle control, and sub-cycle breaker operation.

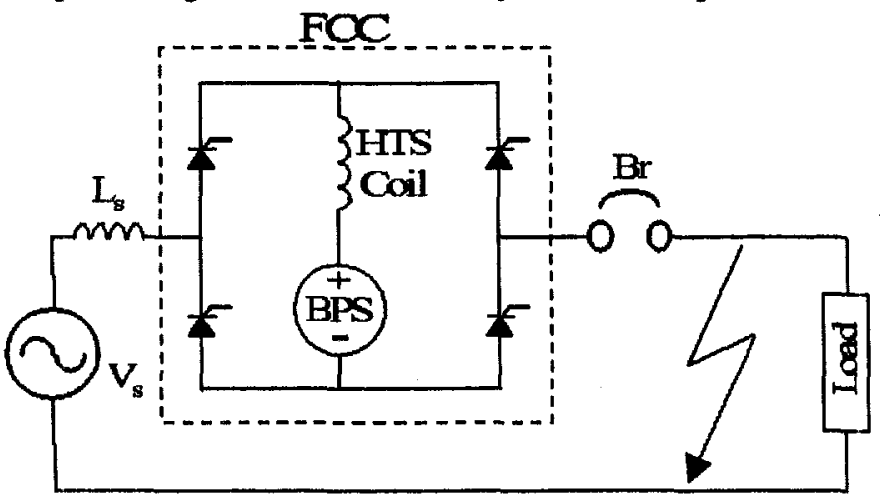

Fig. 1. Schematic showing keys components of FCC and location in typical network. $V_{s}$ is the voltage source, $L_{b}$ is the source inductance, $B r$ is the breaker, and BPS is the bias power supply.

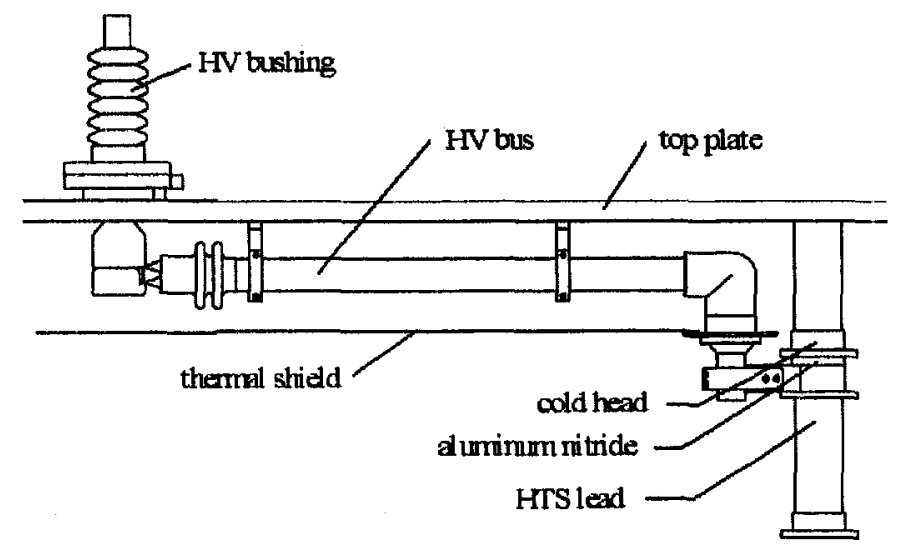

Fig. 2. Drawing of the HV bus, top plate of the vacuum vessel, and the cold head which provides the low temperature thermal intercept for the bus. 


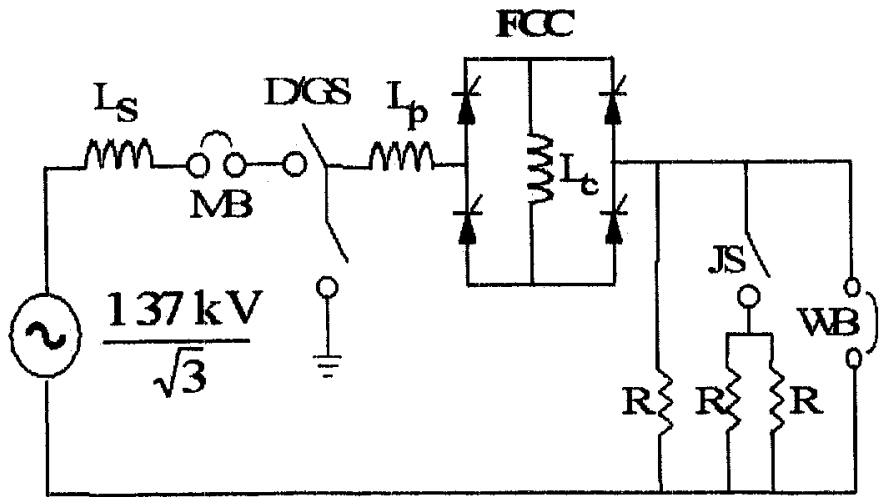

Fig. 3. Substation test circuit for the FCC. The substation includes the $13.7 \mathrm{kV}$ source, the source impedance $L_{8}$ and the master breaker, MB. The disconnect/grounding switch is $D / G S ; L_{p}$ is the protection inductor, $R$ is the load resistance of $64 \Omega$; JS is a Jennings switch, and WB is a Westinghouse breaker.

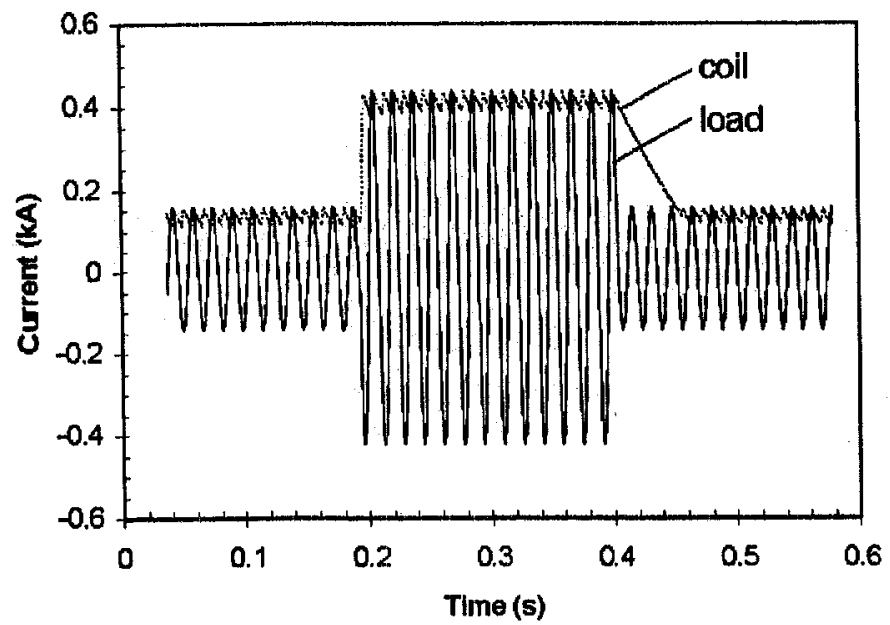

Fig. 4. Illustration of a load step test. The ac load current and the corresponding nearly dc current through the FCC coil are shown for an initial load of $1 \mathrm{MW}$, switching to $3 \mathrm{MW}$ at about $0.2 \mathrm{~s}$ and back to $1 \mathrm{MW}$ at $0.4 \mathrm{~s}$.

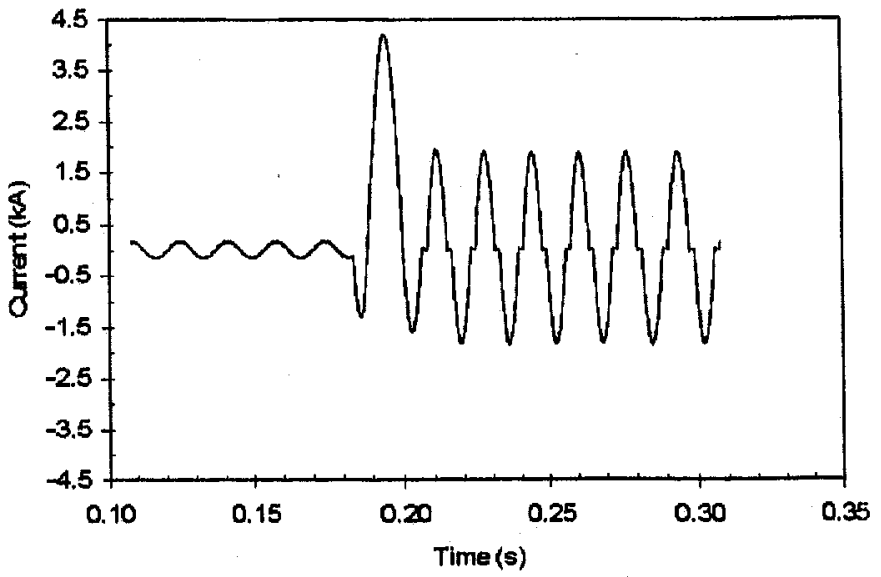

Fig. 5. An example of the response of the FCC to a fault applied at $0.18 \mathrm{~s}$ when the phase delay angle is set for 120 degrees. For times less than $0.18 \mathrm{~s}$, the FCC was responding to a $1 \mathrm{MW}$ load.

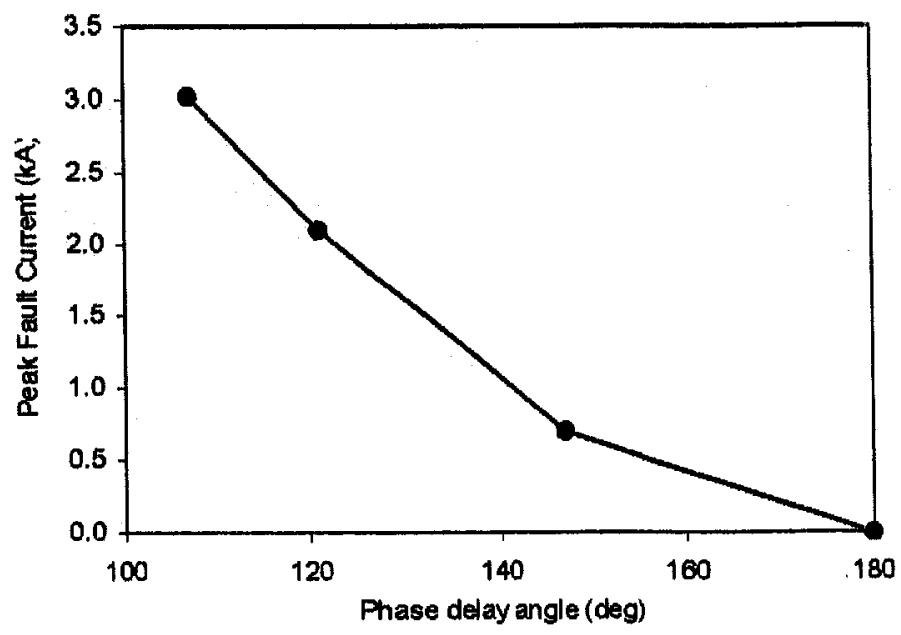

Fig. 6. Four data points connected by straight lines for illustration purposes to demonstrate the dependence of the magnitude of the fault current on the setting of the phase delay angle.

\section{ACKNOWLEDGMENT}

The authors would like to thank Ernie Serna and Darrell Roybal for their excellent work in fabricating the new HV bus and many other components. Their skills in assembling and testing the device were invaluable.

\section{REFERENCES}

[1] E. Leung, et al., "Design \& Development of a $15 \mathrm{kV}, 20 \mathrm{kA}$ HTS Fault Current Limiter," IEEE Transactions on Applied Superconductivity, vol. 10, no. 1, pp832-835, Mar 2000.

[2] H. J. Boenig, C. H. Mielke, B. L. Burley, H. Chen, J. A. Waynert, "The Bridge-Type Fault Controller - a New FACTS Controller," IEEE 2002 PES Summer Meeting Proceedings, submitted for publication.

[3] M. Hara, J. Suehiro, H. Shigematsu, S. Yano, "Methods for the Improvement of Electrical Insulation in Vacuum in the Presence of Transverse Magnetic Field," Electrical Engineering in Japan, vol. 110, no. 2, pp. 27-35, 1990.

[4] M. Hara, H. Shigematsu, S. Yano, K. Yamafuji, M. Takeo, K. Funaki, "Influence of transverse magnetic field on breakdown charcteristics of vacuum, gaseous helium at low temperature and liquid helium," Cryogenics, vol. 29, pp. 448-456, Apr 1989.

[5] A. Neuber, H. Keene, J. Minter, "Surface Flashover Testing of an Aluminum Nitride Disc," IEEE Power Modulator Conference, submitted for publication, July 2002 . 